\title{
MATURIDADE EM INDÚSTRIA 4.0: O CASO DE UMA EMPRESA DE MRO DE MOTORES DE HELICÓPTEROS
}

Fabio Pinheiro de Carvalho Haltenburg (fabiohaltenburg@gmail.com) - Universidade de Brasília/ Faculdade de Tecnologia

Cristiane Monteiro Santos Nogueira (cristiane.monteiro@safrangroup.com) - SAFRAN - SHE Andrea Cristina dos Santos (andreakieck@gmail.com) - Universidade de Brasília/ Faculdade de Tecnologia/ Laboratório Aberto de Brasília

\section{RESUMO}

Este projeto teve como objetivo adaptar um modelo de maturidade de Indústria 4.0 para montadora de motores de helicópteros no Brasil. Para isso, foram estudados os modelos de maturidade, as tecnologias habilitadoras e os princípios de implementação da Quarta Revolução Industrial. A partir da base teórica, foram selecionados quatro modelos de maturidade em Indústria 4.0 para estudo detalhado. Sendo que dois modelos foram aplicados, os resultados obtidos constataram que os modelos eram muito genéricos para promover melhorias na empresa. Foi então desenvolvido um comparativo entre os modelos selecionados para escolher qual deles serviria de base para adaptação. Utilizando-se o método AHP, foram estabelecidos cinco critérios e seus respectivos pesos para ser possível comparar as alternativas. O modelo que obteve o maior valor global foi eleito e modificado para melhor atender às especificidades do caso estudado. A adaptação do modelo selecionado envolve adequação do questionário de avaliação de maturidade, a estrutura de níveis de maturidade e as dimensões e campos associados. O modelo adaptado foi então aplicado e os seus resultados serviram como base para o desenvolvimento de sugestões de melhoria para a empresa.

Palavras chave: modelo de avaliação de maturidade, Indústria 4.0, adaptação, estudo de caso, MRO aeronáutico 


\section{INTRODUÇÃ̃}

A quarta revolução industrial - conhecida como Revolução Digital ou ainda Indústria 4.0 - se baseia em um grupo de novas tecnologias aplicadas em conjunto para obter maior eficiência e atender a novas demandas do mercado. Essas novas tecnologias são conhecidas como tecnologias habilitadoras que quando aplicadas em conjunto, integram o mundo virtual e o físico, possibilitando o trabalho colaborativo de máquinas e humanos com maior eficiência e mínima ociosidade e desperdício.

Neste contexto, a cadeia de valor do setor aeronáutico tem buscado de forma intensa a aplicação das tecnologias habilitadoras para manter-se competitiva no mercado (SAFRANGROUP, 2019). Sendo um setor fortemente regulamentado por órgãos de controle, apresenta padrões de manufatura bem definidos permitindo maior possibilidades de automatização e controle. Contudo, quando são analisadas as etapas finais da cadeia de valor - em especial o pós-venda, que inclui as atividades de manutenção, reparo e revisão geral de componentes, também conhecido como MRO (Maintanece, Repair and Overhaul) - alguns desafios ainda persistem, sendo que nesse caso as muitas regulamentações funcionam como barreiras à implementação de novas tecnologias.

Por outro lado, a indústria de MRO, tem aspecto importante nos negócios, uma vez que apresenta o maior lucro operacional dos fabricantes de equipamentos, devido os serviços do pós-venda (UHLMANN, BILZ e BAUMGARTEN, 2013). Logo, o objetivo de inserir a indústria de MRO no contexto da Revolução Digital é criar empresas capazes de se adaptar continuamente às condições graças ao uso de tecnologias relevantes, aprendizado organizacional e processos de tomada de decisão que obtém vantagens de dados com alta qualidade disponíveis com maior rapidez (SCHUH et al., 2017) .

Para definir a implementação da Indústria 4.0, é necessário definir o estado a ser alcançado, o que torna mais clara a visualização do caminho a ser seguido. O conceito de maturidade está intimamente ligado a esse estado, de modo que a avaliação de maturidade é verificar quão longe uma empresa está de seu estado desejado (EROL e SIHN, 2016). As informações decorrentes dessa avaliação serão usadas para desenvolver planejamentos, reduzindo esforços em projetos de pouco e impacto. Existem, na literatura, diferentes modelos de avaliação de maturidade para indústrias de manufatura. Apesar disso, é necessário adequar a avaliação às diferenças da indústria de MRO em relação à indústria de manufatura. 
Considerando os benefícios que podem ser obtidos a partir da avaliação de maturidade e da existência de mais de um modelo para essa avaliação, o tema do trabalho apresenta-se da seguinte forma: modelos de avaliação de maturidade e como eles podem ser aplicados a um caso.

A empresa estudada, cujo principal negócio é o MRO, desenvolve um projeto para implementação da Indústria 4.0 em seu site e, com isso, poderá obter direcionamento para o seu projeto com a avaliação de sua maturidade. A empresa será alvo do estudo de caso a ser apresentado nessa pesquisa. As especificidades da empresa analisada e a diversidade de modelos de avaliação de maturidade serviram como base para desenvolver a seguinte questão de pesquisa: Como realizar a avaliação de maturidade no caso selecionado?

\section{REVISÃO TEÓRICA}

A Indústria 4.0 por sua vez baseia-se em oito avanços tecnológicos, chamados de tecnologias habilitadoras: robótica adaptativa, big data, simulação, sistemas embarcados, internet das coisas (IoT), sistemas de nuvem, manufatura aditiva e tecnologias de virtualização. Essas tecnologias habilitadoras devem ser suportadas por sete princípios de projeto que permitem a adaptação e coordenação de todo o sistema empresarial. Tecnologias básicas como segurança cibernética, sensores e atuadores, RFID e RTLS e tecnologias móveis também devem dar suporte aos avanços tecnológicos (WANG, WANG, 2016).

Para que a implementação da Indústria 4.0 ocorra de maneira adequada, é importante que sejam seguidos princípios de implementação. Segundo Salkin et al. (2018), existem sete princípios para a implementação da Indústria 4.0 que serão descritos a seguir.

a) Gerenciamento de dados em tempo real: permite a monitoramento do sistema para evitar problemas quando um sistema falha.

b) Interoperabilidade: está relacionada com a comunicação de componentes de sistemas cyber-físicos. O uso da IoT e de processos padronizados auxiliam a construção das fábricas inteligentes.

c) Virtualização: permite monitorar a adaptação de um sistema, mudanças de um sistema ou simplesmente o sistema como um todo por meio de ferramentas de simulação e realidade aumentada. 
d) Descentralização: fundamental para a permitir a tomada de decisões por máquinas que baseiam o seu aprendizado em eventos e ações anteriores.

e) Agilidade: flexibilidade do sistema a mudanças em requisitos, como ao modificar um módulo de um sistema de software padronizado com interfaces de hardware também padronizadas.

f) Orientação ao serviço: é a adaptação do sistema aos requisitos dos clientes a partir da integração dos subsistemas internos e externos.

g) Processos de negócio integrados: liga os sistemas físicos e plataformas de software permitindo comunicação e mecanismos de coordenação auxiliados pelos serviços de gerenciamento de dados e redes conectadas.

As tecnologias habilitadoras fornecem a base para o desenvolvimento da Indústria 4.0 enquanto os princípios de implementação orientam e integram as tecnologias, permitindo que a empresa entre na revolução digital de maneira homogênea.

Os modelos de maturidade são comumente usados como instrumento para capar e medir a maturidade de uma organização em relação a uma determinada meta (EROL E SIHN, 2016). Os modelos incluem dimensões e níveis, de modo que cada dimensão representa capacidades específicas do domínio de interesse e os níveis são rótulos ordinais que significam estágios de maturidade (SANTOS, 2018).

Algumas das vantagens de se utilizar um modelo de maturidade são que ele se encontra pronto para uso, já foram testados e, em muitos casos, continuam a ser desenvolvidos por pessoas e instituições envolvidas. Dentre as desvantagens estão que existem modelos com escopos que atendem somente parcialmente à necessidade, podem ser muito generalistas e não mostrar a dinâmica real da instituição avaliada e são únicos e precisam ser usados na própria forma como foram construídos (SANTOS, 2018).

\section{METODOLOGIA}

Para adaptar um modelo, foram selecionados os seguintes modelos de maturidade em Indústria 4.0 da literatura:

Modelo 1: IMPULS-industrie 4.0-readiness (LICHTBLAU et al., 2018);

Modelo 2: A maturity model for assessing industry 4.0 readiness and maturity of manufacturing enterprises (SCHUMACHER, EROL E SIHN., 2016); 
Modelo 3: Industrie 4.0 Maturity Index (SCHUH et al., 2017).

Modelo 4: Maturity and Readiness Model for Industry 4.0 Strategy (AKDIL; USTUNDAG; CEVIKCAN, 2018)

Após a definição da estrutura conceitual teórica e da compreensão dos modelos selecionados, o desenvolvimento da pesquisa se deu em três etapas: a aplicação dos modelos existentes, a adaptação do modelo de maturidade para o caso e a aplicação desse modelo adaptado. A adaptação do modelo faz parte do planejamento do caso definido por Cauchick (2007) e é a etapa em que serão aplicados os modelos selecionados e uma análise comparativa dos modelos selecionados será desenvolvida.

Para a adaptação do modelo de maturidade, considerou-se as características do site em questão: a atividade principal é o MRO de motores de helicópteros, porém, os modelos mostrados no capítulo de referencial teórico são focados na indústria manufatureira. Os modelos 3 e 4 possuem foco de aplicação abrangente, enquanto os outros dois modelos avaliados - 1 e 2 - possuem área de aplicação limitada. Por isso, a aplicação em uma empresa de MRO precisa ser adaptada, visto que existem tecnologias habilitadoras e princípios da Indústria 4.0 que têm relevância menor para esse tipo de Indústria.

Para realizar a adaptação optou-se por aplicar os modelos cujas perguntas de avaliação estão disponíveis. Nesse caso, os modelos 1 e 4 estavam disponíveis e foram aplicados com o intuito tanto de realizar um teste piloto como de obter avaliações que pudessem ajudar na adaptação do modelo. Além disso, foi feita uma análise comparativa dos modelos existentes selecionados para escolher o modelo que melhor se enquadra para a adaptação e verificar os pontos fortes e fracos dos modelos para a adaptação ao caso estudado.

A adaptação do modelo será o produto da análise de três bloco mostrado na Figura 1: dos modelos de maturidade existentes, da Indústria 4.0 ideal e do estado atual do caso. 
FIGURA 1 - Interseção dos blocos para adaptação do modelo. Fonte: O Autor (2019)

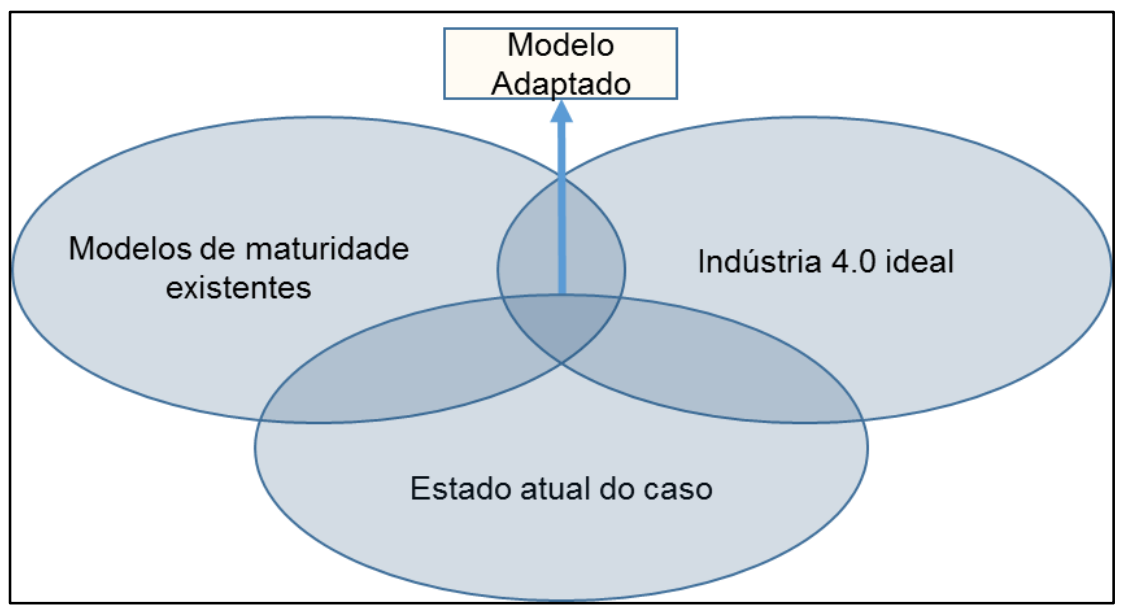

\section{RESULTADOS E DISCUSSÃO}

\subsection{Aplicação dos Modelos de Maturidade}

Os Modelos 1 e 4, respectivamente dos autores Lichtblau et al. (2015) e Akdil, Ustundag e Cevikcan (2018), possuem questionários prontos e disponíveis e por isso puderam ser aplicados para o caso estudado. A aplicação dos questionários facilitou a adaptação do modelo, pois foram a primeira experiência de aplicação de avaliação de maturidade em Indústria 4.0 na empresa. A compreensão dos temas abordados nas avaliações foi melhor, facilitando a posterior aplicação do modelo adaptado.

No Modelo 1 a empresa foi ranqueada no nível 1 de maturidade (Beginner). A pontuação final foi de 1,458 em 5 pontos possíveis. Para cada dimensão, a avaliação dá o respectivo nível e disponibiliza sugestões para melhoria do nível de maturidade. A Figura 2 mostra a distribuição da quantidade de empresas do grupo de comparação nos níveis de maturidade.

A partir do Modelo 4 é apresentado na Figura 3 o gráfico do tipo radar com o resultado da aplicação do questionário. Nas três dimensões a empresa atingiu o Nível 1 - Existência dessa maneira, a avaliação de maturidade da empresa nesse modelo resultou no segundo nível entre quatro possíveis. 
FIGURA 2 - Comparação nível de maturidade geral (Modelo 4). Fonte: O autor (2019)

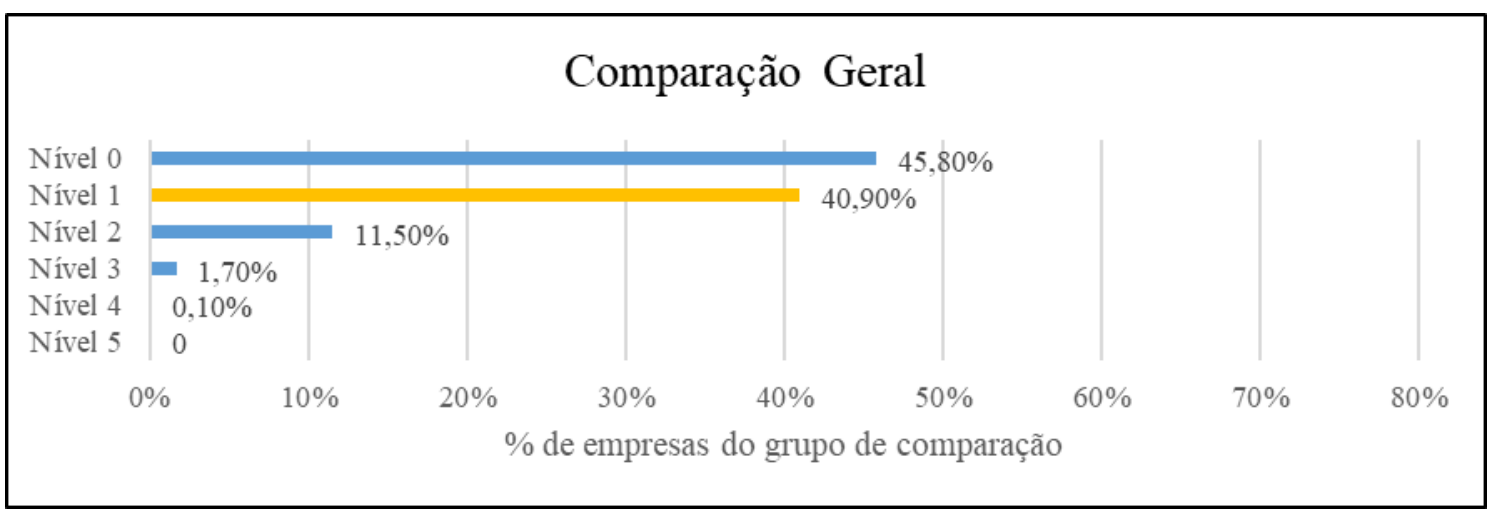

FIGURA 3 - Resultado Geral Modelo 4. Fonte: O Autor (2019)

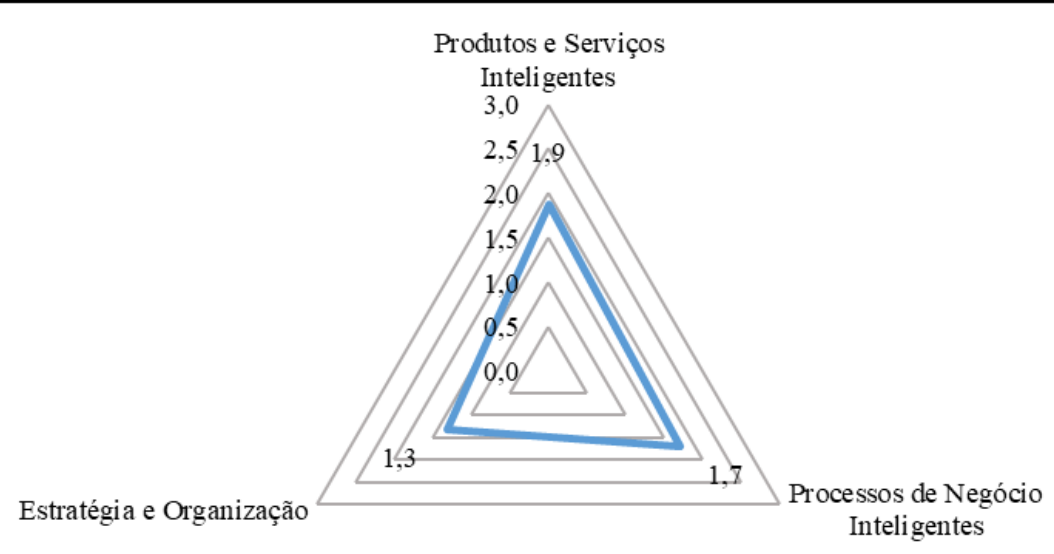

\subsection{Escolha do Modelo para adaptação}

Para comparar as características dos modelos, foi utilizado o método AHP ou análise multicritério. A escolha do método de comparação se deu pelo fato de os critérios para comparação serem subjetivos, de difícil quantificação, mas hierarquizáveis. Dessa maneira, a metodologia para comparação consistirá nas seguintes etapas:

1. Definição explícita dos critérios

2. Determinação da importância relativa de cada critério

3. Avaliação dos critérios para cada alternativa

4. Valoração global de cada alternativa 
Os critérios foram definidos a partir das necessidades observadas pelo autor com base no planejamento de uso do modelo no caso estudado. Critério 1 é o formato de divisão em blocos (dimensões ou áreas estruturais). Nele será avaliado se a quantidade de blocos está em quantidade e nível de descrição suficientes para facilitar o entendimento e dar uma visão detalhada para os tomadores de decisão.

Critério 2: é o nível de detalhamento das perguntas. Para esse critério deve-se avaliar a que nível de detalhamento as perguntas chegam. Não deve ser muito profundo para não afetar o tempo de aplicação, porém o detalhamento superficial pode diminuir a acurácia da avaliação.

Critério 3: é o formato de divisão dos níveis de maturidade. Nesse critério deve ser levado em conta que ter poucos níveis de maturidade leva a ter grandes saltos de um nível para outro, o que pode diminuir a impressão de melhoria após aplicação de novas tecnologias e desenvolvimento de novos projetos na empresa. Por exemplo, caso seja aplicado o modelo em um ano e após dois anos seja aplicado novamente, se houver poucos níveis, será mais difícil o salto de um nível para o outro, mesmo com mudanças significativas na empresa. Portanto, devem existir mais níveis e cada um deles devem ter requisitos explicativos.

Critério 4: é a adaptabilidade. Nesse critério é levada em consideração como o modelo pode ser adaptado para uma realidade diferente. Para facilitar a adaptação, o modelo não deverá ter pesos para determinar as pontuações dos blocos e as perguntas podem ser deixadas em branco sem prejuízo à avaliação da maturidade.

Critério 5: é a facilidade de aplicação. Nesse caso, os outros aspectos têm influência sobre esse critério, pois um modelo com uma quantidade excessiva de questões ou de blocos dificulta a aplicação, assim como a falta de um questionário pronto - algo que ocorre no Modelo 3. Além disso, a disponibilidade do questionário e a automatização do cálculo de maturidade como ocorre no Modelo 1 auxilia a facilidade de aplicação.

Atribuiu-se, então, notas nos critérios para cada um dos modelos e foi feito o cálculo do valor global, como mostrado na Tabela 1 . Nela é possível notar que o Modelo 4 obteve o maior valor global e por isso foi o modelo escolhido como base para o caso. 
TABELA 1 - Valor global comparação entre modelos de maturidade

\begin{tabular}{|c|c|c|c|c|c|c|c|}
\hline & \multicolumn{5}{|c|}{ Critérios $(\mathbf{C j})$} & \multirow{2}{*}{$\begin{array}{c}\text { Valor global } \\
\text { (V) }\end{array}$} \\
\hline & & 1 & 2 & 3 & 4 & 5 & \\
\hline \multirow{4}{*}{ 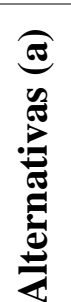 } & Modelo 1 & 9 & 3 & 9 & 3 & 9 & 4,89 \\
\hline & Modelo 2 & 7 & 8 & 4 & 5 & 4 & 5,79 \\
\hline & Modelo 3 & 9 & 6 & 9 & 7 & 3 & 6,63 \\
\hline & Modelo 4 & 4 & 9 & 5 & 9 & 7 & 7,86 \\
\hline
\end{tabular}

Com os resultados dos valores globais e da avaliação dos critérios, observa-se que os modelos 1 e 3 destacam-se no seu formato de divisão em blocos (Critério 1), apesar de fazerem isso de maneira distinta. No Modelo 1 são definidas seis dimensões com descrição detalhada, enquanto no Modelo 3 são definidas quatro áreas estruturais que são analisadas em cinco áreas funcionais também detalhadamente descritas. O Modelo 4 possui três dimensões descritas de maneira superficial.

Sob o ponto de vista do Critério 2, nível de detalhamento das perguntas, os Modelos 4 e 2 são os líderes, pois ambos possuem grande número de perguntas para a avaliação e consequentemente podem trazer perguntas mais detalhadas sobre a situação da empresa. $\mathrm{O}$ modelo 1 é um destaque negativo pois possui menor número questões, mostrando ser um modelo mais superficial. O Modelo 3 por não possuir um questionário feito pelos autores, deixa o detalhamento das perguntas à escolha do aplicador do modelo e por isso fica em um nível intermediário.

Para o Critério 3, formato de divisão dos níveis de maturidade, os Modelos 1 e 3 são os que obtiveram melhor avaliação dividirem a maturidade em seis níveis detalhadamente explicados. A explicação para os cinco níveis de maturidade do Modelo 2 é demasiadamente superficial e o Modelo 4 apesar de apresentar explicação detalhada para cada nível, define apenas quatro níveis.

Observando as avaliações das alternativas no Critério 4, a adaptabilidade, nota-se que os modelos 3 e 4 possuem as maiores notas. O Modelo 4 possui a melhor adaptabilidade pois não determina peso para as suas perguntas o que permite excluir ou incluir perguntas compatíveis com a avaliação proposta. O Modelo 3 não possui perguntas definidas, o que 
facilita a adaptabilidade, porém não foi pensado como sendo um modelo adaptável, o que o torna restrito à realidade para qual foi criado. O Modelo 2 não prevê adaptabilidade e apesar de o questionário dos autores não ser acessível, há um modelo a ser seguido. O Modelo 1 é o que encontra mais barreiras para a adaptabilidade, pois o cálculo da maturidade é baseado na importância relativa de cada dimensão que foi determinada a parti da aplicação dos questionários em um certo número de empresas.

O Critério 5, a facilidade de aplicação, também é produto dos outros critérios e, observando a avaliação desse critério, percebe-se que o Modelo 1 é o de mais fácil aplicação por possuir um formulário online no qual a avaliação pode ser feita em questão de minutos e o resultado é dado instantaneamente ao fim do preenchimento. $\mathrm{O}$ modelo 4 não possui formulários online, mas disponibiliza o questionário completo. Ainda sobre o Modelo 4, algumas perguntas deveriam ter melhor explicação para avaliação pois não seguem um padrão como indicado para o Modelo 3 ou o Modelo 2. O modelo 3 tem a pior avaliação por não possuir um questionário pronto, obrigando o aplicador a desenvolver as próprias questões.

A partir dos resultados da aplicação dos Modelos 1 e 4 e da análise dos Modelos 3 e 4, e do comparativo entre os modelos selecionados, as características do modelo proposto foram divididas em:

- Dimensões

- Níveis de maturidade

- Questionário

Definiu-se cinco dimensões, seis níveis de maturidade e 64 questões distribuídas pelas dimensões. Os resultados da comparação entre os modelos existentes indicaram que o Modelo 4 é o que melhor se adequada como base para a adaptação e os pontos positivos dos outros modelos serão o guia para as modificações, assim como as características do caso.

Para comparar o Modelo Adaptado aos outros modelos analisados, o método AHP foi aplicado da mesma maneira que foi utilizado para a seleção do melhor modelo para base da adaptação. Para facilitar a comparação, os critérios e pesos apontados serão os mesmos. A Tabela 2 mostra o valor global atingido pelo Modelo Adaptado (M. A.) quando comparado aos outros modelos. 
Para o cálculo da maturidade das dimensões e da empresa como um todo, serão utilizadas as Equações (1) e (2) nas quais M é a maturidade, D é dimensão, Q é a avaliação atribuída a cada questão, $\mathrm{m}$ é o número de questões, n é o número de dimensões e $\mathrm{G}$ é usado para se referir à empresa como um todo.

$$
\begin{aligned}
& M_{D j}=\sum_{i=1}^{m} \frac{Q_{D i}}{m} \\
& M_{G}=\sum_{j=1}^{n} \frac{M_{D j}}{n}
\end{aligned}
$$

\begin{tabular}{|c|c|c|c|c|c|c|c|}
\hline & & \multicolumn{5}{|c|}{ Critérios (Cj) } & \multirow{2}{*}{$\begin{array}{c}\text { Valor global } \\
\text { (V) }\end{array}$} \\
\hline & & 1 & 2 & 3 & 4 & 5 & \\
\hline \multirow{5}{*}{ 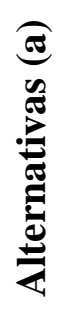 } & Modelo 1 & 9 & 3 & 9 & 3 & 9 & 4,89 \\
\hline & Modelo 2 & 7 & 8 & 4 & 5 & 4 & 5,79 \\
\hline & Modelo 3 & 9 & 6 & 9 & 7 & 3 & 6,63 \\
\hline & Modelo 4 & 4 & 9 & 5 & 9 & 7 & 7,86 \\
\hline & M. A. & 9 & 9 & 9 & 9 & 7 & 8,75 \\
\hline
\end{tabular}

TABELA 2 - Valor global comparação com o Modelo Adaptado

Os resultados para os valores globais mostram que o modelo adaptado agregou as melhores características dos modelos analisados. A exceção é a facilidade de aplicação que não alcançou o nível determinado pelo Modelo 1. O motivo para isso é a falta de uma plataforma de aplicação online do modelo, característica que, se aplicada na adaptação, ultrapassaria o escopo do presente trabalho. A Tabela 3 mostra os principais pontos positivos de cada modelo analisado que foram incorporados ao Modelo Adaptado.

TABELA 3 - Pontos positivos incorporados ao Modelo Adaptado

\begin{tabular}{cccc}
\hline Modelo 1 & Modelo 2 & Modelo 3 & Modelo 4 \\
\hline Formato de divisão & Forma de pontuação & Formato das & Questionário; \\
em blocos; & final; & perguntas; & Sub-dimensões; \\
\hline
\end{tabular}


A aplicação do modelo adaptado mostrou que o valor da maturidade geral da empresa é 2,50 - nível Intermediário - e que as cinco dimensões obtiveram as pontuações como mostrado na FIGURA . Pelo fato de os Modelos 1 e 4 já terem sido aplicados anteriormente, a aplicação do novo modelo tornou-se mais simples, especialmente se for considerada a equivalência com as questões do Modelo 4. A seguir serão apresentados os resultados da aplicação do modelo proposto.

FIGURA 4 - Resultados da aplicação do Modelo Adaptado. Fonte: O Autor (2019)

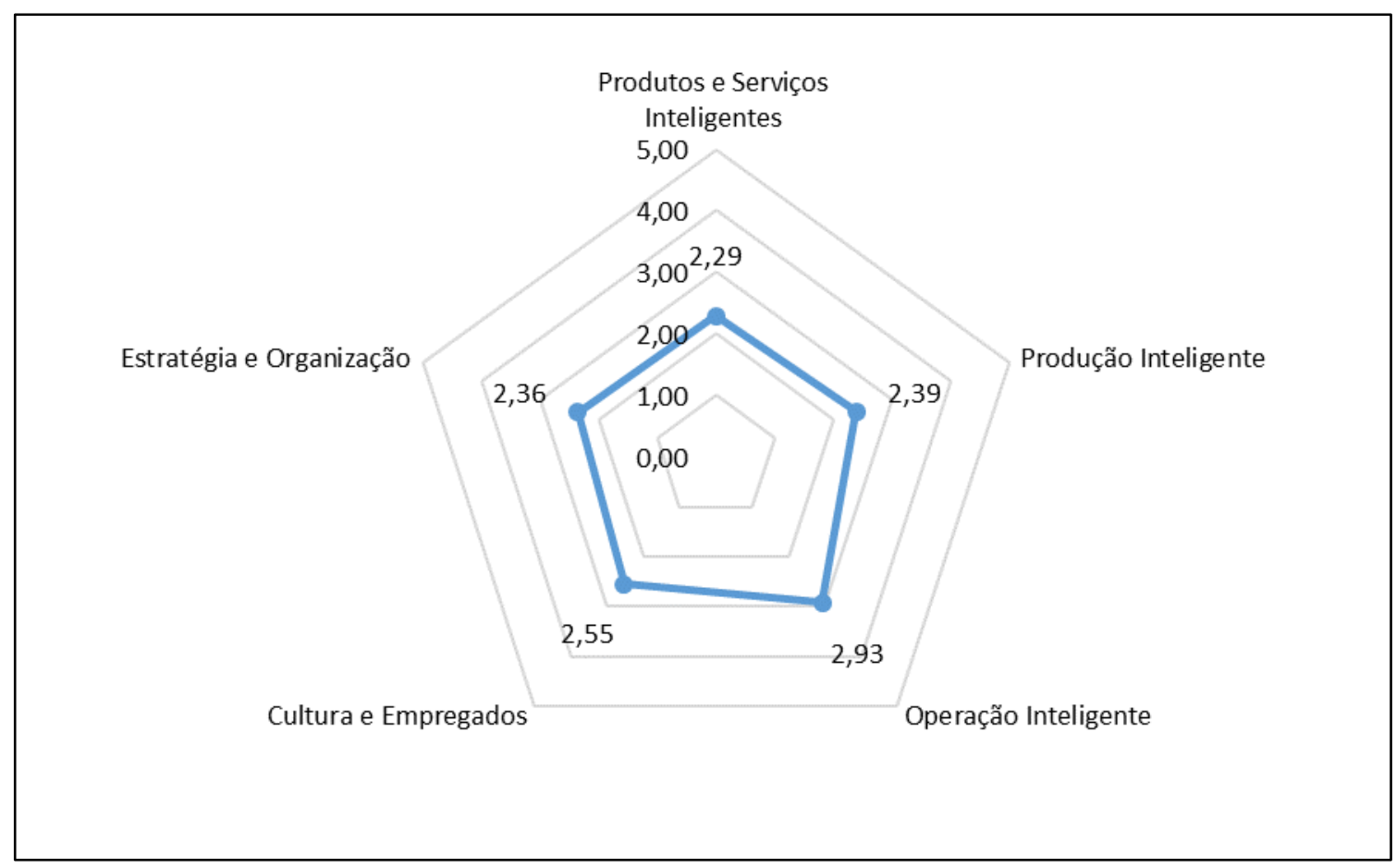

Ao comparar a aplicação dos Modelos 1 e 4 com a do Modelo Adaptado são observadas diferenças consideráveis entre os resultados. Por ser um modelo com questões em menor quantidade e mais superficiais e foco na manufatura, a pontuação obtida no Modelo 1 colocou a empresa avaliada em uma posição inferior no nível de maturidade em relação ao Modelo Adaptado (a comparação entre eles pode ser feita pelo fato de os níveis de maturidade serem congruentes). Em relação ao Modelo 4, a diferença de pontuação precisa ser analisada com atenção, pois é necessário verificar a equivalência entre os níveis de maturidade. Ao realizar essa operação, a equivalência da pontuação obtida no Modelo 4 - 1,3 - seria atualizada para 
2,16 considerando uma nota de variando de 0 a 5 ao invés de 0 a 3 . Assim, observa-se que a pontuação é similar à encontrada no Modelo Adaptado.

\section{CONCLUSÃO}

A inserção de uma empresa na Indústria 4.0 não é algo corriqueiro e tão pouco irrelevante para as empresas que pretendem manter ou aumentar o seu nível de competitividade. A aplicação das tecnologias habilitadoras, assim como o uso dos princípios de implementação formam a base necessária para o desenvolvimento de soluções que fazem parte da Revolução Digital. Avaliar o quanto a empresa está alinhada com essas soluções - realizar a avaliação da maturidade em Indústria 4.0 - é importante. Para empresas de manufatura o uso dos modelos de maturidade existentes já é suficiente para avaliar a maturidade, porém a falta de uma referência que consiga avaliar todas as características do MRO e da empresa estudada mostrou que a adaptação de um modelo para avaliação de maturidade deveria tornar-se o foco desse trabalho.

Para a adaptação, foi necessário compreender o relacionamento entre os as tecnologias habilitadoras, os princípios de aplicação da Indústria 4.0 e os modelos de maturidade. Além disso, selecionou-se quatro modelos de maturidade existentes para então serem comparados por critérios definidos para a adaptação para escolher qual seria o melhor como base para a adaptação. O comparativo permitiu obter pontos positivos de cada modelo e utilizar esses pontos no desenvolvimento do Modelo Adaptado. A adaptação ocorreu com base no Modelo 4 que obteve melhor pontuação. As três dimensões originais do Modelo 4 foram transformadas em cinco, enquanto que os níveis de maturidade foram modificados para ficarem análogos aos do Modelo 1. O questionário do modelo adaptado utilizou as questões do Modelo 4, porém adaptadas para um formato similar ao do Modelo 3, cujos níveis de maturidade são análogos às opções de resposta.

Com os resultados da aplicação do modelo em conjunto com a análise de cada dimensão foi possível determinar pontos de melhoria. A empresa poderá utilizar os resultados como guia para os próximos passos da implementação da Indústria 4.0, de modo que cada modificação em sua maturidade possa ser determinada com o uso do Modelo Adaptado. 
O Modelo Adaptado, além de ter sido aplicado ao caso estudado, também poderia analisar a maturidade de outras empresas cujo modelo de negócio seja o MRO. Isso porque o Modelo Adaptado desenvolvido nesse projeto prioriza os pontos mais relevantes dentro de um negócio de MRO. Os modelos existentes são voltados, de modo geral, para a manufatura e por isso foi necessária a alteração no Modelo 4 em campos como o desenvolvimento de produtos - que não ocorre dentro de um site que faz somente $\mathrm{MRO}$ - e o serviço de pós-venda, que nesse caso pode ser agregado ao desenvolvimentos de produtos de modo geral.

\section{REFERÊNCIAS}

AKDIL, Kartal Yagiz; USTUNDAG, Alp; CEVIKCAN, Emre. Maturity and Readiness Model for Industry 4.0 Strategy. Em: Industry 4.0: Managing The Digital Transformation. Springer, Cham, 2018. p. 61-94.

CAUCHICK, P. A. Estudo de caso na engenharia de produção: estrutura e recomendações para a sua condução. Produção, 17 (1), 216-229. 2007.

LICHTBLAU, K. et al. IMPULS-industrie 4.0-readiness. Impuls-Stiftung des VDMA, Aachen-Köln, 2015.

SAFRAN-GROUP. 2018 Registration Document. Disponível em <https://www.safrangroup.com/sites/group/files/safran_ddr_2018_uk.pdf> Acesso em: 02 de maio de 2019.

SALKIN, Ceren et al. A Conceptual Framework for Industry 4.0. In: Industry 4.0: Managing The Digital

Transformation. Springer, Cham, 2018. p. 3-23.

SANTOS, Reginaldo Carreiro. Proposta de modelo de avaliação de maturidade da indústria 4.0. 2018. Tese de Doutorado.

SCHUMACHER, Andreas; EROL, Selim; SIHN, Wilfried. A maturity model for assessing industry 4.0 readiness and maturity of manufacturing enterprises. Procedia CIRP, v. 52, p. 161-166, 2016.

SCHUH, Günther et al. Industrie 4.0 Maturity Index. Managing the Digital Transformation of Companies. Munich: Herbert Utz, 2017.

UHLMANN, Eckart; BILZ, Martin; BAUMGARTEN, Jeannette. MRO-challenge and chance for sustainable enterprises. Procedia Cirp, v. 11, p. 239-244, 2013.

WANG, Lidong; WANG, Guanghui. Big data in cyber-physical systems, digital manufacturing and industry 4.0. International Journal of Engineering and Manufacturing (IJEM), v. 6, n. 4, p. 1-8, 2016. 